\title{
On Inverse Nodal Problem and Multiplicities of Eigenvalues of a Vectorial Sturm-Liouville Problem
}

\author{
Xiaoyun Liu \\ School of Mathematics and Physics, Anyang Institute of Technology, Anyang, 455000 Henan, China \\ Correspondence should be addressed to Xiaoyun Liu; xyl.hb@163.com
}

Received 19 December 2019; Revised 20 May 2020; Accepted 26 May 2020; Published 2 July 2020

Academic Editor: Raúl E. Curto

Copyright (C) 2020 Xiaoyun Liu. This is an open access article distributed under the Creative Commons Attribution License, which permits unrestricted use, distribution, and reproduction in any medium, provided the original work is properly cited.

An $m$-dimensional vectorial inverse nodal Sturm-Liouville problem with eigenparameter-dependent boundary conditions is studied. We show that if there exists an infinite sequence $\left\{\mathbf{y}_{n_{j}, r}\left(x, \lambda_{n_{j}}^{2}, r\right)\right\}_{j=1}^{\infty}$ of eigenfunctions which are all vectorial functions of type (CZ), then the potential matrix $Q(x)$ and $A$ are simultaneously diagonalizable by the same unitary matrix $U$. Subsequently, some multiplicity results of eigenvalues are obtained.

\section{Introduction}

Consider the following $m$-dimensional vectorial SturmLiouville problem with the eigenparameter-dependent boundary conditions:

$$
\left\{\begin{array}{l}
-\mathbf{y}^{\prime \prime}+Q(x) \mathbf{y}=\lambda^{2} \mathbf{y}, 0 \leq x \leq \pi \\
\mathbf{y}(0)=\mathbf{0} \\
A \mathbf{y}^{\prime}(\pi)+\lambda \mathbf{y}(\pi)=\mathbf{0}
\end{array}\right.
$$

where $\lambda$ is the spectral parameter and $\mathbf{y}=\left(y_{1}, y_{2}, \cdots, y_{m}\right)^{T}$ is an $m$-dimensional vectorial function. The potential matrix $Q(x)$ is an $m \times m$ real symmetric and nonnegative definite matrix-valued function which is defined and integrable on the interval $[0, \pi] . A$ is an $m \times m$ nonsingular real symmetric matrix. Throughout this paper, we set $m \geq 2$ and agree that $\mathbf{0}$ denotes the $m$-dimensional zero vector.

Sturm-Liouville problems with eigenparameter in the boundary conditions arise upon separation of variables in the one-dimensional wave and heat equations for various physical applications [1]. There are some literatures on such scalar problems (see [2-5]) and vectorial problems (see [6$8]$ ). We consider the inverse nodal problem of (1) firstly. It was McLaughlin who initiated the study of the inverse nodal problem [9]. In the recent years, the inverse nodal problem of the Sturm-Liouville problem has been investigated a lot (see the monographs [10-17] and the references therein) and the vectorial inverse nodal problem is studied in $[18,19]$. However, McLaughlin's uniqueness theorem does not hold for the vectorial Sturm-Liouville problems. To clarify our problem, the following definitions could be seen in $[18,19]$. For the convenience of reader, we state here again. Let $\mathbf{y}(x)$ be an $m$-dimensional vectorial function defined on the interval $[0, \pi]$, if $\mathbf{y}\left(x_{0}\right)=\mathbf{0}, x_{0} \in[0, \pi], x_{0}$ will be called a nodal point (zero) of $\mathbf{y}(x)$. We say that $\mathbf{y}(x)$ is a vectorial function of type (CZ) (or $\mathbf{y}(x)$ has a common zero property) if all the isolated zeros of its components are nodal points. The matrix $Q(x)$ is called simultaneously diagonalizable if there is a constant unitary matrix $U$ such that $U^{*} Q(x) U$ is a diagonal matrixvalued function.

In 1999, Shen and Shieh [18] studied the inverse nodal problem of the vectorial equation in (1) with Dirichlet boundary condition when dimension $m=2$. They proved that if the problem has infinitely many eigenfunctions $\left\{\mathbf{y}_{n_{j}}(x)\right\}_{j=1}^{\infty}$ which are of type (CZ), then $Q(x)$ is simultaneously diagonalizable. It seems to be the first study of the vectorial inverse nodal problem. Cheng et al. generalized to the arbitrary separated boundary conditions in [19]. Chan [6] investigated some eigenvalue problems of (1). He proved 
that the eigenvlaues of the problem are all real. Based on the results of $[6,18,19]$, in this study, using a different method from that in $[18,19]$, we consider the inverse nodal problem of (1). Firstly, we derived that the matrix $\int_{0}^{x} Q(t) d t$ is simultaneously diagonalizable which is equivalent to $Q(x)$ possessing the same property. That is, there exists a constant unitary matrix $U$ such that $U^{*} Q(x) U=\operatorname{diag}\left(\mu_{1}(x), \mu_{2}(x)\right.$, $\left.\cdots, \mu_{m}(x)\right)$, where $\mu_{i}(x)(i=1,2, \cdots, m)$ are eigenvalues of $Q(x)$. Meanwhile, if $Q(x)$ and $A$ are simultaneously diagonalizable by the same unitary matrix $U$, we prove that the eigenfunctions of problem (1) are all of type (CZ) (see Corollary 3.2).

Next, we investigate the relationship between multiplicities of eigenvalues of problem (1) and matrix $A$. It is known that the eigenvalues of a scalar Sturm-Liouville problem with the separate boundary conditions are all simple. However, the multiplicities of the $m$-dimensional vectorial SturmLiouville problem may be among 1 to $m$. Such problems were studied in [20-22]. In [20], Shen and Shieh study the multiplicities of 2-dimensional vectorial Sturm-Liouville problem defined in $[0,1]$. Suppose that $Q(x)=\left(q_{i j}(x)\right)$ is a continuous $2 \times 2$ Jacobian matrix-valued function; it is proved that if $\int_{0}^{1}$ $q_{12}(x) d x \neq 0$, then the sufficiently large eigenvalues are simple. Subsequently, Kong [21] developed and improved the results in [20] to the case when $Q(x)$ is real symmetric. In 2007, Yang et al. [22] extended the result of [20,21] to the Sturm-Liouville equation with a weighted function $w$, a leading coefficient function $p$, and general separated conditions.

From the estimation of eigenvalues (see Lemma 4), it is not difficult to see that the multiplicities of eigenvalues of problem (1) are determined firstly by the multiplicities of eigenvalues of matrix $A$ no matter $Q(x)$ possesses any properties. We provide conditions on $A$ and $Q(x)$ under which, with finitely many exceptions, the eigenvalues of vectorial problem (1) are simple.

This paper is divided into four sections. Following this Introduction, in Section 2, we investigate the asymptotic expression of the eigenvalues and state some other preliminary lemmas for the main theorems. In Section 3, we discuss the inverse nodal problem of (1). In Section 4, some results about multiplicities of eigenvalues of the 2-dimensional vectorial Sturm-Liouville problem (1) are given.

\section{Preliminaries}

Let $Y\left(x, \lambda^{2}\right)$ be the matrix solutions of equation

$$
-Y^{\prime \prime}+Q(x) Y=\lambda^{2} Y, \quad x \in[0, \pi]
$$

satisfying the initial conditions

$$
\begin{aligned}
Y\left(0, \lambda^{2}\right) & =0_{m}, \\
Y^{\prime}\left(0, \lambda^{2}\right) & =E_{m},
\end{aligned}
$$

where $E_{m}$ denotes the $m \times m$ identity matrix and $0_{m}$ denotes the $m \times m$ zero matrix. Denote

$$
\Delta(\lambda)=\operatorname{det}\left(A Y^{\prime}\left(\pi, \lambda^{2}\right)+\lambda Y\left(\pi, \lambda^{2}\right)\right) .
$$

$\Delta(\lambda)$ will be called the characteristic function of the eigenvalues of problem (1). The algebraic multiplicity of an eigenvalue $\lambda$ is the order of $\lambda$ as a zero of $\Delta(\lambda)$. The geometric multiplicity of $\lambda$ as an eigenvalue of the problem (1) is defined to be the number of linearly independent solutions of the boundary problem. Let $C(\lambda)=A Y^{\prime}\left(\pi, \lambda^{2}\right)+\lambda Y\left(\pi, \lambda^{2}\right)$.

Lemma 1. The geometric multiplicity of $\lambda$ as an eigenvalue of problem (1) is equal to $m-\operatorname{Rank}(C(\lambda))$.

From the above lemma, the geometric multiplicity of an eigenvalue $\lambda_{*}$ of problem (1) is at most $m$, and the geometric multiplicity of $\lambda_{*}$ is $m$ if and only if $C\left(\lambda_{*}\right)$ is the zero matrix. It is known that $\Delta(\lambda)$ is an entire function of order one with respect to $\lambda$. What is more, when $Q(x)$ is nonnegative definite for any $x \in[0, \pi]$, the eigenvalues of problem (1) are all real [6]. Denote $\lambda=\sigma+i \tau, \sigma, \tau \in \mathbb{R}$. By variation of constants, we have

$$
Y\left(x, \lambda^{2}\right)=\frac{\sin (\lambda x)}{\lambda} E_{m}+\frac{1}{\lambda} \int_{0}^{x} \sin \lambda(x-t) Q(t) Y\left(t, \lambda^{2}\right) d t .
$$

Lemma 2 ([23]). For fixed $x \in[0, \pi]$, the matrix functions $Y\left(x, \lambda^{2}\right)$ and $Y^{\prime}\left(x, \lambda^{2}\right)$ are entire functions with respect to variable $\lambda$. When $|\lambda| \longrightarrow \infty$, we have

$$
\begin{gathered}
Y\left(x, \lambda^{2}\right)=\frac{\sin (\lambda x)}{\lambda} E_{m}+O\left(\frac{e^{|\tau| x}}{|\lambda|^{2}}\right), \\
Y^{\prime}\left(x, \lambda^{2}\right)=\cos (\lambda x) E_{m}+O\left(\frac{e^{|\tau| x}}{|\lambda|}\right) .
\end{gathered}
$$

Remark 3. Using the first relation in (6) to iterate equation (5), we find that

$$
\begin{aligned}
Y\left(x, \lambda^{2}\right)= & \frac{\sin (\lambda x)}{\lambda} E_{m}-\frac{\cos (\lambda x)}{2 \lambda^{2}} \int_{0}^{x} Q(t) d t \\
& +\frac{1}{2 \lambda^{2}} \int_{0}^{x} \cos \lambda(x-2 t) Q(t) d t+O\left(\frac{e^{|\tau| x}}{|\lambda|^{3}}\right) .
\end{aligned}
$$

By Riemann-Lebesgue lemma,

$$
\lim _{\lambda \rightarrow \infty} \int_{0}^{x} \cos \lambda(x-2 t) Q(t) d t=0 .
$$

Thus, when $|\lambda| \longrightarrow \infty, Y\left(x, \lambda^{2}\right)$ has the following asymptotic expansion:

$$
Y\left(x, \lambda^{2}\right)=\frac{\sin (\lambda x)}{\lambda} E_{m}-\frac{\cos (\lambda x)}{2 \lambda^{2}} \int_{0}^{x} Q(t) d t+o\left(\frac{e^{|\tau| x}}{|\lambda|^{2}}\right) .
$$


Similarly,

$$
Y^{\prime}\left(x, \lambda^{2}\right)=\cos (\lambda x) E_{m}+\frac{\sin (\lambda x)}{2 \lambda} \int_{0}^{x} Q(t) d t+o\left(\frac{e^{|\tau| x}}{|\lambda|}\right)
$$

Lemma 4. Suppose that an eigenvalue $\lambda_{n, r}(r=1,2, \cdots, m)$ is with algebraic multiplicity $k(1 \leq k \leq m)$. When $|n| \longrightarrow \infty$, $\lambda_{n, r}$ have the following asymptotic expansion:

$$
\lambda_{n, r}=n-\frac{1}{\pi} \tan ^{-1} a_{r}+O\left(\frac{1}{n^{1 / k}}\right)
$$

where $a_{r}$ are eigenvalues of matrix $A$.

Proof. Since $A$ is an $m \times m$ nonsingular real symmetric matrix, there exists a unitary matrix $U$ such that

$$
U^{*} A U=\operatorname{diag}\left(a_{1}, a_{2}, \cdots, a_{m}\right)
$$

where $a_{1} \leq a_{2} \leq \cdots \leq a_{m}$. By formula (6) in Lemma 2,

$$
\begin{aligned}
U^{*} C(\lambda) U= & \operatorname{diag}\left(a_{1}, a_{2}, \cdots, a_{m}\right) \cos (\lambda \pi) \\
& +\sin (\lambda \pi) E_{m}+O\left(\frac{e^{|\tau| \pi}}{|\lambda|}\right) .
\end{aligned}
$$

Thus,

$$
\begin{aligned}
\Delta(\lambda) & =\operatorname{det} C(\lambda)=\operatorname{det} U^{*} C(\lambda) U \\
& =\Pi_{r=1}^{m}\left(a_{r} \cos (\lambda \pi)+\sin (\lambda \pi)\right)+O\left(\frac{e^{m|\tau| \pi}}{|\lambda|}\right) \\
& =\Pi_{r=1}^{m} R_{r} \sin \left(\lambda \pi+\theta_{r}\right)+O\left(\frac{e^{m|\tau| \pi}}{|\lambda|}\right)
\end{aligned}
$$

where $R_{r}=\left(a_{r}^{2}+1\right)^{1 / 2}, \theta_{r}=\tan ^{-1} a_{r}$ and $\left|\theta_{r}\right|<\pi / 2$. Additionally, $\theta_{1} \leq \theta_{2} \leq \cdots \leq \theta_{m}$. Denote $\Delta_{0}(\lambda)=\Pi_{r=1}^{m} R_{r} \sin \left(\lambda \pi+\theta_{r}\right)$ and $\delta(\lambda)=O\left(e^{m|\tau| \pi} /|\lambda|\right)$. Take a closed $\lambda$-curve $\Gamma_{n}=\Gamma_{n}^{(1)} \cup$ $\Gamma_{n}^{(2)} \cup \Gamma_{n}^{(3)}(n=1,2, \cdots)$ :

$$
\begin{aligned}
\Gamma_{n}^{(1)} & =\left\{\lambda=\sigma+i \tau\left|\sigma=n+\frac{1}{2}-\frac{\theta_{1}}{\pi}, 0 \leq\right| \tau \mid \leq n\right\}, \\
\Gamma_{n}^{(2)} & =\left\{\lambda=\sigma+i \tau\left|\sigma=-\left(n+\frac{1}{2}\right)-\frac{\theta_{m}}{\pi}, 0 \leq\right| \tau \mid \leq n\right\}, \\
\Gamma_{n}^{(3)} & =\left\{\lambda=\sigma+i \tau\left|-\left(n+\frac{1}{2}\right)-\frac{\theta_{m}}{\pi} \leq \sigma \leq n+\frac{1}{2}-\frac{\theta_{1}}{\pi},\right| \tau \mid=n\right\} .
\end{aligned}
$$

$$
\begin{aligned}
& \text { On } \Gamma_{n}^{(1)} \text { or } \Gamma_{n}^{(2)}, \text { for } r=1,2, \cdots, m, \\
& \begin{aligned}
\left|\sin \left(\lambda \pi+\theta_{r}\right)\right| & =\left|\frac{1}{2 i}\left(e^{i\left(\sigma \pi+\theta_{r}\right)} e^{-\tau \pi}-e^{-i\left(\sigma \pi+\theta_{r}\right)} e^{\tau \pi}\right)\right| \\
& \geq \frac{e^{\tau \pi}}{2}\left(1-\left|e^{2 i\left(\sigma \pi+\theta_{r}\right)} e^{-2 \tau \pi}\right|\right)>\frac{e^{|\tau| \pi}}{4} .
\end{aligned}
\end{aligned}
$$

On $\Gamma_{n}^{(3)}$, similarly, we have $\left|\sin \left(\lambda \pi+\theta_{r}\right)\right|>e^{|\tau| \pi} / 4$ for $r=1,2, \cdots, m$. Denote $M=\Pi_{r=1}^{m}\left|R_{r}\right|$. On the contour $\Gamma_{n}$, since $|\lambda| \geq 1$, we have

$$
\left|\Delta_{0}(\lambda)\right|_{\Gamma_{n}}=\left|\Pi_{r=1}^{m} R_{r} \sin \left(\lambda \pi+\theta_{r}\right)\right|_{\Gamma_{n}}>M \cdot \frac{e^{m|\tau| \pi}}{4^{m} \cdot|\lambda|}
$$

that is, $\left|\Delta_{0}(\lambda)\right|_{\Gamma_{n}}>|\delta(\lambda)|_{\Gamma_{n}}$. Using Rouché theorem, the number of zeros of $\Delta(\lambda)$ inside $\Gamma_{n}$ coincides with the number of zeros of $\Delta_{0}(\lambda)$; i.e., it equals to $(2 n+1) m$ (counting the multiple zeros). Note that the zeros of $\Delta(\lambda)$ and $\Delta_{0}(\lambda)$ are all real. Now, we consider the zeros of $\Delta_{0}(\lambda)$ in $\Gamma_{n}$. They are

$$
\lambda_{j, r}^{0}=j-\frac{\theta_{r}}{\pi}, j=0, \pm 1, \cdots, \pm n, r=1,2, \cdots, m
$$

Now, it is easy to see that the algebraic multiplicity of an eigenvalue $\lambda$ of problem (1) is between 1 to $m$. Note that the eigenvalues of problem (1), in this case, stretch from $-\infty$ to $+\infty$. Consequently, we denote all the eigenvalues as $\lambda_{n, r}$ $(n=0, \pm 1, \pm 2, \cdots ; r=1,2, \cdots, m)$. For sufficiently large $n$, $\lambda_{n, r}$ lies between $\Gamma_{n-1}$ and $\Gamma_{n}$. Set $\lambda_{n, r}=\lambda_{n, r}^{0}+\varepsilon_{n, r}=n-\left(\theta_{r}\right)$ $\pi)+\varepsilon_{n, r}$. Moreover, denote $C_{\delta}^{n, r}:=\left\{\lambda:\left|\lambda-\lambda_{n, r}^{0}\right| \leq \delta\right\}$, where $0<\delta<\min _{1 \leq i, j \leq m, i \neq j}\left\{(1 / \pi)\left|\theta_{i}-\theta_{j}\right|\right\}$. Applying the Rouché theorem again to the circle $C_{\delta}^{n, r}$, if $\lambda_{n, r}^{0}$ is $k$-multiple $(1 \leq k$ $\leq m)$, in $C_{\delta}^{n, r}$, there are $k$ zeros of $\Delta(\lambda)$. For sufficiently small $\delta$, it follows that $\varepsilon_{n, r}=o(1)$.

If $\lambda_{n, r}$ is $k$-multiple, substituting $\lambda_{n, r}$ into (14) and noting that $\tau=0$, we get that $\left(\varepsilon_{n, r}\right)^{k}=O(1 / n)$. Consequently, $\varepsilon_{n, r}=$ $O\left(1 / n^{1 / k}\right)$.

Using the asymptotic expression of eigenvalues, we get that the asymptotic expression about the nodal points.

Lemma 5. Suppose that $\mathbf{y}_{n, r}\left(x, \lambda_{n, r}^{2}\right)$ is an eigenfunction of the vectorial problem (1) of type (CZ) corresponding to the eigenvalue $\lambda_{n, r}$, and $\left\{x_{k}^{n, r}\right\}$ is the nodal set of $y_{n, r}\left(x, \lambda_{n, r}^{2}\right)$. Then,

(i) for sufficiently large $|n|, \mathbf{y}_{n, r}\left(x, \lambda_{n, r}^{2}\right)$ possesses $|n|$ nodal points $x_{k}^{n, r}$ in $(0, \pi)$

(ii) the nodal points have the following asymptotic expression:

$$
x_{k}^{n, r}=\frac{(k+1) \pi}{|n|}+O\left(\frac{1}{n^{2}}\right), k=0,1, \cdots,|n|-1,
$$

for sufficiently large $|n|$

(iii) $\left\{x_{k}^{n, r}: 0 \leq k \leq|n|-1\right\}_{|n|=1}^{\infty}$ is dense in $[0, \pi]$

Proof. The proof is similar to that of Lemma 2 in [18] and Theorem 2.3 in [19]. 


\section{Inverse Nodal Problem}

In this section, we discuss the inverse nodal problem of (1).

Theorem 6. Suppose that there exists a sequence $\left\{\boldsymbol{y}_{n_{j}, r}\left(x, \lambda_{n_{j}, r}^{2}\right)\right\}_{j=1}^{\infty}$ of eigenfunctions of problem (1) which are all vectorial functions of type $(C Z)$, then $Q(x)$ and $A$ are simultaneously diagonalizable by the same unitary matrix $U$.

Proof. Denote $\mathbf{v}_{n_{j}, r}$ a unit null vector of $C\left(\lambda_{n_{j}, r}\right)$, where $Y$ $\left(x, \lambda_{n_{j}, r}^{2}\right)$ is the solution of matrix initial problem (2)-(3) corresponding to $\lambda_{n_{j}, r}$. Then,

$$
\mathbf{y}_{n_{j}, r}\left(x, \lambda_{n_{j}, r}^{2}\right)=Y\left(x, \lambda_{n_{j}, r}^{2}\right) \mathbf{v}_{n_{j}, r}
$$

is an eigenfunction of vectorial problem (1) corresponding to $\lambda_{n_{j}, r}$. Assuming that $\mathbf{y}_{n_{j}, r}\left(x, \lambda_{n_{j}, r}^{2}\right)$ is a vectorial eigenfunction of type (CZ), by Lemma 5, we know that, $\mathbf{y}_{n_{j}, r}$ $\left(x, \lambda_{n_{j}, r}^{2}\right)$ has $\left|n_{j}\right|$ nodal points $x_{k}^{n_{j}, r}$ in $(0, \pi)$. What is more, $\left\{x_{k}^{n_{j}, r}|k=0,1, \cdots,| n_{j} \mid-1\right\}_{j=1}^{\infty}$ is dense in $[0, \pi]$; thus for a fixed $x_{0}$ in $(0, \pi]$, there exists a sequence of nodal $x_{k_{j}}^{n_{j}, r}$ such that

$$
\lim _{j \rightarrow \infty} x_{k_{j}}^{n_{j}, r}=x_{0}
$$

Since

$$
\mathbf{y}_{n_{j}, r}\left(x_{k_{j}}^{n_{j}, r}, \lambda_{n_{j}, r}^{2}\right)=Y\left(x_{k_{j}}^{n_{j}, r}, \lambda_{n_{j}, r}^{2}\right) \mathbf{v}_{n_{j}, r}=\mathbf{0},
$$

by (9), we have

$$
\begin{aligned}
Y\left(x_{k_{j}}^{n_{j}, r}, \lambda_{n_{j}, r}^{2}\right)= & \frac{\sin \left(\lambda_{n_{j}, r} x_{k_{j}}^{n_{j}, r}\right)}{\lambda_{n_{j}, r}} E_{m} \\
& -\frac{\cos \left(\lambda_{n_{j}, r} x_{k_{j}, r}^{n_{j}, r}\right)}{2 \lambda_{n_{j}, r}^{2}} \int_{0}^{x_{k_{j}}^{n_{j}, r}} Q(t) d t \\
& +o\left(\frac{1}{\left|\lambda_{n_{j}, r}\right|^{2}}\right) .
\end{aligned}
$$

Thus,

$$
\int_{0}^{x_{k_{j}}^{n_{j}, r}} Q(t) d t \cdot \mathbf{v}_{n_{j}, r}=\frac{2 \lambda_{n_{j}, r} \sin \left(\lambda_{n_{j}, r} x_{k_{j}}^{n_{j}, r}\right)}{\cos \left(\lambda_{n_{j}, r} x_{k_{j}}^{n_{j}, r}\right)} E_{m} \cdot \mathbf{v}_{n_{j}, r}+o(1) \cdot \mathbf{v}_{n_{j}, r} .
$$

Since $\left\|\mathbf{v}_{n_{j}, r}\right\|=1, v_{n_{j}, r}$ converges to some unit vector denoting as $\mathbf{v}$. Therefore, taking the limit as $j \longrightarrow \infty$ for both sides of Equation (24), we obtain

$$
\int_{0}^{x_{0}} Q(t) d t \cdot \mathbf{v}=c_{1}\left(x_{0}\right) \cdot \mathbf{v}
$$

where $c_{1}\left(x_{0}\right)$ is a scalar associated with $x_{0}$. Since $x_{0}$ is arbitrary in $(0, \pi]$, we can choose $x_{1} \neq x_{0}$ in $(0, \pi]$ and use the above argument for $x_{1}$ such that $\mathbf{v}$ is also an eigenvector of $\int_{0}^{x_{1}} Q(t) d t$. Then, $\int_{0}^{x_{0}} Q(t) d t$ and $\int_{0}^{x_{1}} Q(t) d t$ have the same eigenvector $\mathbf{v}$. That is, $\mathbf{v}$ is chosen independently of $x$.

Furthermore, using Schmidt orthogonalization, $\mathbf{v}$ could expand into standard orthogonal basis denoting as $\mathbf{u}_{1}, \mathbf{u}_{2}$, $\cdots, \mathbf{u}_{m}$. Since $\int_{0}^{x} Q(t) d t$ is diagonalizable for all $x \in(0, \pi]$, $\mathbf{u}_{2}, \mathbf{u}_{3}, \cdots, \mathbf{u}_{m}$ have to be eigenvectors for $\int_{0}^{x} Q(t) d t$. Thus, there are other scalars $c_{i}(x)(i=2,3, \cdots, m)$ depending on $x$ such that $\int_{0}^{x} Q(t) d t \cdot \mathbf{u}_{i}=c_{i}(x) \cdot \mathbf{u}_{i}$. Hence, we derive that $\int_{0}^{x} Q(t) d t$ is simultaneously diagonalizable. In fact, denote $U=\left[\mathbf{u}_{1}, \mathbf{u}_{2}, \cdots, \mathbf{u}_{m}\right]$, then we have

$$
U^{*} \int_{0}^{x} Q(t) d t U=\operatorname{diag}\left(c_{1}(x), c_{2}(x), \cdots, c_{m}(x)\right) .
$$

Deriving the two sides of the above formula, we derived that matrix $Q(x)$ is also simultaneously diagonalizable by the same matrix $U$. That is,

$$
U^{*} Q(x) U=\Lambda(x)=\operatorname{diag}\left(\mu_{1}(x), \mu_{2}(x), \cdots, \mu_{m}(x)\right),
$$

where $\mu_{i}(x)=c_{i}^{\prime}(x)(i=1,2, \cdots, m)$.

Since $\mathbf{y}_{n_{j}, r}\left(x, \lambda_{n_{j}, r}^{2}\right)$ satisfies the boundary conditions, hence,

$$
A Y^{\prime}\left(\pi, \lambda_{n_{j}, r}^{2}\right) \mathbf{v}_{n_{j}, r}+\lambda_{n_{j}, r} Y\left(\pi, \lambda_{n_{j}, r}^{2}\right) \mathbf{v}_{n_{j}, r}=\mathbf{0} .
$$

By Lemma 2, we have

$$
A \mathbf{v}_{n_{j}, r}=-\tan \left(\lambda_{n_{j}, r} \pi\right) \mathbf{v}_{n_{j}, r}+O\left(\frac{1}{n_{j}}\right) .
$$

Let $j \longrightarrow \infty, \mathbf{v}_{n_{j}, r}$ tends to the same vector $\mathbf{v}$, and the sequence $\tan \left(\lambda_{n_{j}, r} \pi\right)$ has a limit $-c \in \mathbb{R}$. Thus, $A \mathbf{v}=c \mathbf{v}$. Subsequently, with a similar process, we conclude that $A$ is $\operatorname{diag}$ onalizable by the same matrix $U$. That is,

$$
U^{*} A U=\Lambda_{A}=\operatorname{diag}\left(a_{1}, a_{2}, \cdots, a_{m}\right) .
$$

The proof of the theorem is finished.

Theorem 7. Suppose that $Q(x)$ and $A$ are simultaneously diagonalizable by the same unitary matrix $U$. Then,

(1) the eigenfunctions of problem (1) are all of type (CZ)

(2) the algebraic multiplicities and geometric multiplicities of eigenvalues of problem (1) are equal 
Proof.

(1) By the transformation $\mathbf{y}=U \mathbf{z}$, the problem becomes

$$
\begin{aligned}
\mathbf{z}^{\prime \prime} & =U^{-1} \mathbf{y}^{\prime \prime}=U^{-1}\left(Q U \mathbf{z}-\lambda^{2} U \mathbf{z}\right), \mathbf{z}(0) \\
& =\mathbf{0}, \Lambda_{A} \mathbf{z}^{\prime}(\pi)+\lambda \mathbf{z}(\pi)=\mathbf{0},
\end{aligned}
$$

that is,

$$
-\mathbf{z}^{\prime \prime}+\Lambda(x) \mathbf{z}=\lambda^{2} \mathbf{z}, \mathbf{z}(0)=\mathbf{0}, \Lambda_{A} \mathbf{z}^{\prime}(\pi)+\lambda \mathbf{z}(\pi)=\mathbf{0} .
$$

Note that the two problems (1) and (32) have exactly the same eigenvalues and multiplicities. Let $\mathbf{z}=$ $\left(z_{1}, z_{2}, \cdots, z_{m}\right)^{T}$. The vectorial problem (32) is equivalent to the following scalar Sturm-Liouville problems:

$$
\left\{\begin{array}{l}
-z_{i}^{\prime \prime}+\mu_{i}(x) z_{i}=\lambda^{2} z_{i}, \quad x \in[0, \pi] \\
z_{i}(0)=0 \\
a_{i} z_{i}^{\prime}(\pi)+\lambda z_{i}(\pi)=0 \\
i=1,2, \cdots, m
\end{array}\right.
$$

All the eigenvalues of problem (32) are the collections of eigenvalues of scalar problems in (33). If $\lambda_{*}^{(i)}$ is an eigenvalue of the $i$ th problem in (33) and $z_{*, i}\left(x, \lambda_{*}^{(i)}\right)$ is the eigenfunction corresponding to $\lambda_{*}^{(i)}$, then

$$
\mathbf{y}_{*}\left(x, \lambda_{*}^{(i)}\right)=U\left(0, \cdots, z_{*, i}\left(x, \lambda_{*}^{(i)}\right), \cdots, 0\right)^{T}
$$

is the eigenfunction of the original problem (1) corresponding to $\lambda_{*}^{(i)}$. Obviously, $\mathbf{y}_{*}\left(x, \lambda_{*}^{(i)}\right)$ is a vectorial function of type (CZ).

(2) Let $z_{0, i}\left(x, \lambda^{2}\right)(i=1,2, \cdots, m)$ be the solutions of equations in (33) satisfying the initial conditions

$$
z_{0, i}\left(0, \lambda^{2}\right)=0, z_{0, i}^{\prime}\left(0, \lambda^{2}\right)=1
$$

We have

$$
\begin{aligned}
C(\lambda)= & \operatorname{diag}\left(a_{1} z_{0,1}^{\prime}\left(\pi, \lambda^{2}\right)+\lambda z_{0,1}\left(\pi, \lambda^{2}\right), a_{2} z_{0,2}^{\prime}\left(\pi, \lambda^{2}\right)\right. \\
& \left.+\lambda z_{0,2}\left(\pi, \lambda^{2}\right), \cdots, a_{m} z_{0, m}^{\prime}\left(\pi, \lambda^{2}\right)+\lambda z_{0, m}\left(\pi, \lambda^{2}\right)\right) .
\end{aligned}
$$

Thus, $\Delta(\lambda)=\Pi_{i=1}^{m}\left(a_{i} z_{0, i}^{\prime}\left(\pi, \lambda^{2}\right)+\lambda z_{0, i}\left(\pi, \lambda^{2}\right)\right)$. If $\lambda_{*}$ is a $k$-multiple (geometric) eigenvalue, then $\operatorname{Rank}\left(C\left(\lambda_{*}\right)\right)=m-k$. We may as well let the last $k$ elements in the diagonal of $C\left(\lambda_{*}\right)$ be zero. Hence, $\lambda_{*}$ is an eigenvalue of the last $k$ problems in (33). Denote $\Delta_{i}(\lambda)=a_{i} z_{0, i}^{\prime}\left(\pi, \lambda^{2}\right)+\lambda z_{0, i}\left(\pi, \lambda^{2}\right)$.
We have $\Delta_{i}\left(\lambda_{*}\right)=0$ and $\Delta_{i}^{\prime}\left(\lambda_{*}\right) \neq 0$ for $i=m-k+1, \cdots, m$. Thus, $\lambda_{*}$ is also with algebraic multiplicity $k$.

\section{Multiplicities of Eigenvalues}

In this section, we first discuss the relationship between the multiplicities of eigenvalues of 2-dimensional vectorial Sturm-Liouville problem (1) and the matrix $A$ in the boundary condition.

Lemma 8. When $m=2$, the algebraic multiplicity and geometric multiplicity of $\lambda$ as an eigenvalue of the problem (1) is equal.

Proof. See [6].

Theorem 9. Let $m=2$. If the eigenvalues of matrix $A$ are all simple, then with finitely many exceptions, the eigenvalues of problem (1) are all simple too.

Proof. Let $\lambda_{n, r}$ be an eigenvalue of problem (1). By Lemma 4,

$$
\lambda_{n, r}=n-\frac{1}{\pi} \tan ^{-1} a_{r}+o(1) .
$$

Suppose, to the contrary, that the multiplicities of the eigenvalues of problem (1) are 2. By Lemma $1, \operatorname{Rank}(C(\lambda))$ $=0$. That is, $C\left(\lambda_{n, r}\right)=O_{2}$. By (13), we have

$\cos \left(\lambda_{n, r} \pi\right) \operatorname{diag}\left(a_{1}, a_{2}\right)+\sin \left(\lambda_{n, r} \pi\right) E_{2}+O\left(\frac{e^{|\tau|} \pi}{\left|\lambda_{n, r} \pi\right|}\right)=O_{2}$.

Calculating directly and noting that the eigenvalues are real, we get

$$
\tan \left(\lambda_{n, r} \pi\right) E_{2}=\operatorname{diag}\left(a_{1}, a_{2}\right)+O\left(\frac{1}{n}\right) .
$$

Taking the limit as $n \longrightarrow \infty$, (39) follows that $a_{1}=a_{2}$ which contradicts with the facts that the eigenvalues of the matrix $A$ are all simple.

The next theorem shows the relationship between multiplicities of eigenvalues and $Q(x)$ when $m=2$ and $A=c E_{2}$ $(c \neq 0)$.

Theorem 10. Suppose that $A=c E_{2}(c \neq 0)$. If the eigenvalues of the matrix $\int_{0}^{\pi} Q(t) d t$ have no repeated eigenvalues, then, with finitely many exceptions, the eigenvalues of problem (1) are all simple.

Proof. The proof of this conclusion is analogous to that of Theorem 9 and therefore is omitted. Note that formulas (9) and (10) are needed to calculate $C(\lambda)$ in this case.

\section{Data Availability}

No data were used to support the study. 


\section{Conflicts of Interest}

The authors declare that they have no conflicts of interest.

\section{Acknowledgments}

The work is supported by the National Scientific Foundation of China (Grant Nos. 11801012 and 11771017) and the Inner Mongolia Natural Science Foundation (Grant No. 2017MS0125).

\section{References}

[1] C. T. Fulton, "Two-point boundary value problems with eigenvalue parameter contained in the boundary conditions," Proceedings of the Royal Society of Edinburgh, vol. 77, no. 3-4, pp. 293-308, 1977.

[2] H. Hochstadt, "On inverse problems associated with secondorder differential operators," Acta Mathematica, vol. 119, pp. 173-192, 1967.

[3] J. Walter, "Regular eigenvalue problems with eigenvalue parameter in the boundary condition," Mathematische Zeitschrift, vol. 133, no. 4, pp. 301-312, 1973.

[4] P. A. Binding, P. J. Browne, and B. A. Watson, "Inverse spectral problems for Sturm-Liouville equations with eigenparameter dependent boundary conditions," Journal of the London Mathematical Society, vol. 62, no. 1, pp. 161-182, 2000.

[5] P. A. Binding, P. J. Browne, and B. A. Watson, "Sturm-Liouville problems with boundary conditions rationally dependent on the eigenparameter, II," Journal of Computational and Applied Mathematics, vol. 148, no. 1, pp. 147-168, 2002.

[6] C. H. Chan, "Some eigenvalue problems for vectorial SturmLiouville equations with eigenparameter dependent boundary conditions," Transactions of the American Mathematical Society, vol. 364, no. 1, pp. 119-136, 2012.

[7] D. Katar, M. Olgun, and C. Coskun, "Matrix Sturm-Liouville operators with boundary conditions dependent on the spectral parameter," Journal of Nonlinear Sciences and Applications, vol. 9, no. 2, pp. 435-442, 2016.

[8] M. Olgun, "Non-selfadjoint matrix Sturm-Liouville operators with eigenvalue-dependent boundary conditions," Hacettepe Journal of Mathematics and Statistics, vol. 3, no. 1063, pp. 607-614, 2015.

[9] J. R. McLaughlin, "Inverse spectral theory using nodal points as data-A uniqueness result," Journal of Differential Equations, vol. 73, no. 2, pp. 354-362, 1988.

[10] Y. Guo and G. Wei, "Inverse problems: dense nodal subset on an interior subinterval," Journal of Differential Equations, vol. 255, no. 7, pp. 2002-2017, 2013.

[11] C. F. Yang, X. C. Xu, and S. Buterin, "Solution to the interior transmission problem using nodes on a subinterval as input data," Nonlinear Analysis: Real World Applications, vol. 35, no. 35, pp. 20-29, 2017.

[12] G. Freiling and V. Yurko, Inverse Sturm-Liouville Problems and Their Applications, NOVA Science Publishers, New York, 2001.

[13] V. Yurko, "Inverse nodal problems for Sturm-Liouville operators on star-type graphs," Journal of Inverse and Ill-Posed Problems, vol. 16, no. 7, pp. 715-722, 2008.
[14] Y. P. Wang, C. T. Shieh, and H. Y. Miao, "Inverse transmission eigenvalue problems with the twin-dense nodal subset," Journal of Inverse and Ill-Posed Problems, vol. 25, no. 2, pp. 715722, 2017.

[15] S. A. Buterin and C. T. Shieh, "Inverse nodal problem for differential pencils," Applied Mathematics Letters, vol. 22, no. 8, pp. 1240-1247, 2009.

[16] M. Y. Ignatiev and C. T. Shieh, "Recovering the SturmLiouville operator with singular potential using nodal data," Results in Mathematics, vol. 57, no. 1-2, pp. 183-194, 2010.

[17] X. Chen, Y. H. Cheng, and C. K. Law, "Reconstructing potentials from zeros of one eigenfunction," Transactions of the American Mathematical Society, vol. 363, no. 9, pp. 48314851, 2011.

[18] C. L. Shen and C. T. Shieh, "An inverse nodal problem for vectorial Sturm-Liouville equations," Inverse Problems, vol. 16, no. 2, pp. 349-356, 2000.

[19] Y. H. Cheng, C. T. Shieh, and C. K. Law, "A vectorial inverse nodal problem," Proceedings of the American Mathematical Society, vol. 133, no. 5, pp. 1475-1484, 2005.

[20] C. L. Shen and C. T. Shieh, "On the multiplicity of eigenvalues of a vectorial Sturm-Liouville differential equations and some related spectral problems," Proceedings of the American Mathematical Society, vol. 127, no. 10, pp. 2943-2953, 1999.

[21] Q. Kong, "Multiplicities of eigenvalues of a vector-valued Sturm-Liouville problem," Mathematika, vol. 49, no. 1-2, pp. 119-127, 2002.

[22] C. F. Yang, Z. Y. Huang, and X. P. Yang, "The multiplicity of spectra of a vectorial Sturm- Liouville differential equation of dimension two and some applications," Rocky Mountain Journal of Mathematics, vol. 37, no. 4, pp. 1379-1398, 2007.

[23] C. L. Shen, "Some inverse spectral problems for vectorial Sturm-Liouville equations," Inverse Problems, vol. 17, no. 5, pp. 1253-1294, 2001. 